\title{
Robust Fuzzy Sliding Mode Controller for Discrete Nonlinear Systems
}

\author{
Hafedh Abid, Mohamed Chtourou, Ahmed Toumi
}

\begin{abstract}
In this work we are interested to discrete robust fuzzy sliding mode control. The discrete SISO nonlinear uncertain system is presented by the TakgiSugeno type fuzzy model state. We recall the principle of the sliding mode control theory then we combine the fuzzy systems with the sliding mode control technique to compute at each sampling time the control law. The control law comports two terms: equivalent control law and switching control law which has a high frequency. The uncertainty is replaced by its upper bound. Inverted pendulum and mass spring dumper are used to check performance of the proposed fuzzy robust sliding mode control scheme.
\end{abstract}

Keywords: Nonlinear systems, Sliding mode, T-S fuzzy systems, Reaching law.

\section{Introduction}

Many of the industrial plants include nonlinearities or/and uncertainties. To reach the wanted performances, using the classical theories, nonlinearities must be identified to calculate the appropriate controller. The robust control theories is one of the techniques that permits to reach the desired performances in presence of external or/ and internal disturbances. In addition to the stability, the tracking problem must be solved independently of uncertainties. In the literature, many methods have been developed in continuous as well in discrete-time to solve the tracking problem for nonlinear systems. In the last decade many researches combine classical techniques with intelligent one, such as sliding mode with Neuronal systems or sliding mode with fuzzy systems [3][4], to benefit from the advantages of the two control techniques.

The sliding mode control (SMC) was originally developed for variable structure systems in continuous domain. Utkin [12] gives a thorough description of the sliding mode theory in continuous time. Also, Slotine and $\mathrm{Li}[16]$ describe in detail continuous sliding mode controllers.

At the end of the twentieth century, the research of discrete time SMC has been attracted more attention, such as [6],[8],[9],[10], as for the implementation of the controller on a digital computer requires a sampling time and the assumption of an infinite switching time does not hold any more.

The fuzzy systems have been combined with classical sliding mode control to provide robust stability to the fuzzy controller. The combination of the two control principles, is called fuzzy sliding mode control (FSMC), it provides an alternative to design a robust controller for nonlinear systems with uncertainties [15],[14]. Our contribution in this work consists in presenting a new robust fuzzy sliding mode controller based on the Takagi-Sugeno fuzzy state model for discrete nonlinear systems. This paper is organised as follow. In Section 2, we recall the discrete Takagi-Sugeno type fuzzy state model for nonlinear systems. Then, we describe the sufficient and necessary reaching conditions of sliding mode control for discrete nonlinear systems in the first part of the third section ten, a fuzzy sliding mode controller for discrete time of nonlinear systems is developed in the second part and tracking robust fuzzy sliding mode control law is described in the third part. The simulation results of two nonlinear systems show performances of the proposed FSMC in Section 4. Conclusions are drawn in the final section. 


\section{Problem Statement and Fuzzy Systems}

\subsection{Problem Statement}

Consider a class of discrete nonlinear SISO systems described by the following equations:

$$
\left\{\begin{array}{c}
x_{1}(k+1)=x_{2}(k) \\
x_{i}(k+1)=x_{i+1}(k) \\
x_{n}(k+1)=f(X(k))+g(X(k)) u(k) \\
y(k)=C X(k)
\end{array}\right.
$$

where, $\mathrm{C}=[1,0, \ldots, 0], X(k)=\left[x_{1}(k) \quad \ldots \quad . \quad x_{n}(k)\right]^{T} \in R^{n}$ it is the state vector that is assumed to be observable.

We note that $f(X(k))$ and $g(X(k))$ represent two discrete bounded nonlinear functions of the nonlinear SISO systems . They can be obtained from the continuous form by the first order discretized system using Eulers approximation. In order for (1) to be controllable, it is required that $g(X(k)) \neq 0$. If both functions $f(X(k))$ and $g(X(k))$ in (1) are available for feedback, the feedback linearization control can be used to design a well-defined controller, which is usually given in the form:

$$
u(k)=\frac{1}{g(X(k))}\left(-K^{T} E(k)-f(X(k))+x_{n d}(k+1)\right)
$$

where, the state vector $X(k)$ and the desired state vector $X_{d}(k)$ are defined as:

$$
X(k)=\left[\begin{array}{lllll}
x_{1}(k) & . & . & x_{n}(k)
\end{array}\right]^{T} \in R^{n} ; X_{d}(k)=\left[\begin{array}{lllll}
x_{1 d}(k) & . & . & x_{n d}(k)
\end{array}\right]^{T} \in R^{n}
$$

$E(k)$ represents the state tracking error, it is defined as: $E(k)=X(k)-X_{d}(k)$, the vector $K(k)=$ $\left[\begin{array}{lllll}k_{n} & k_{n-1} & . & k_{1}\end{array}\right]^{T} \in R^{n}$ will be chosen such that all roots of the following polynomial $h(s)=$ $s^{n}+k_{1} s^{n-1}+\ldots+k_{n}$ are situated inside the unit complex disc. In general case, the functions $f(X(k))$ and $g(X(k))$ are badly known nonlinear functions so, the control law (2) cannot be implanted. To overcome this difficulty, many approaches are used such as adaptive control, linearization around operating points, fuzzy control... etc.

\subsection{Discrete Takagi-Sugeno type Fuzzy Systems}

The advantage of the T-S type fuzzy models is that their description permits the utilization of the state representation, and by consequence to exploit the maximum of the potential relative to this representation. The Takagi-Sugeno (T-S) type fuzzy model can be viewed as a natural expansion of piecewise linear partition for nonlinear systems. The nonlinear system is represented as a collection of the fuzzy IF-THEN rules, where each rule describes the local dynamics by a linear system model. The general fuzzy model is achieved by fuzzy amalgamation of the linear systems models [1][2]. The $i^{t h}$ rule of the discrete fuzzy model has the following form:

$i^{\text {th }}$ plant rule :

$$
\text { IF } Z_{1} \text { is } \mu_{i 1} \text { and ... and } Z_{n} \text { is } \mu_{\text {in }} \operatorname{THENX}(k+1)=\left(A_{d i}+\Delta A_{d i}\right) X(k)+\left(B_{d i}+\Delta B_{d i}\right) u(k)
$$

where $\left\{\mu_{i j}\right\}$ are the fuzzy sets, $A_{d i} \in R^{n x n}$ and $B_{d i} \in R^{n x m}$ are recpectively the $i^{\text {th }}$ state matrix and the input matrix, $c$ is the number of the IF-THEN fuzzy rules; $u(k)$ is the input vector, $Z(k)=$ $\left[Z_{1}(k)\right.$. . . $\left.Z_{n}(k)\right]$ are the premise variables they represent some measurable system variables, they can be chosen as a state variables. For each rule $R_{i}$ is attributed a weight $w_{i}(z(k))$ which depends on the grade of the membership function of the premise variables $z_{j}(k)$ in fuzzy sets $\mu_{i j}$ : 
$w_{i}(Z(k))=\prod_{j=1}^{n} \mu_{i j}\left(Z_{j}(k)\right) ; w_{i}(Z(k)) \succ 0$; for $i=1, \ldots, c ; \quad \sum_{i=1}^{c} w_{i}(Z(k)) \succ 0$.

where, $\mu_{i j}\left(Z_{j}(k)\right)$ is the grade of the membership function of $Z_{j}(k)$ to the fuzzy set $\mu_{i j}$.

The discrete Takagi-Sugeno type fuzzy model is inferred as follows:

$$
X(k+1)=\frac{\sum_{i=1}^{c} w_{i}(Z(k))\left(\left(A_{d i}+\Delta A_{d i}\right) X(k)+\left(B_{d i}+\Delta B_{d i}\right) u(k)\right)}{\sum_{i=1}^{c} w_{i}(Z(k))}
$$

The normalized weight is defined as which is presented as follow:

$h_{i}(Z(k))=\frac{w_{i}(Z(k))}{\sum_{i=1}^{c} w_{i}(Z(k))} ; 0 \prec h_{i}(Z(k)) \prec 1 ; i=1, \ldots, c ; \sum_{i=1}^{c} h_{i}(Z(k))=1$.

The output of the discrete Takagi-Sugeno type fuzzy model for the uncertain nonlinear systems can be inferred as:

$$
\left\{\begin{array}{c}
X(k+1)=\sum_{i=1}^{c} h_{i}(Z(k))\left(\left(A_{d i}+\Delta A_{d i}\right) X(k)+\left(B_{d i}+\Delta B_{d i}\right) u(k)\right) \\
y(k)=\sum_{i=1}^{c} h_{i}(Z(k)) C_{i} X(k)
\end{array}\right.
$$

where $C_{i}=[1,0,0, \ldots, 0]$.

It is required that all $B_{d i}(k)$ are different from zero to assure the controllability of (5).

\section{Sliding mode control law and reaching conditions}

Sliding mode control, first appeared in the Soviet literature, it has been widely recognized as a potential approach to uncertain dynamical non-linear systems that are subject to external disturbances and parameter variations [12].

In sliding mode control (SMC), the control action forces the system trajectories to cross a manifold of the state space which is called the sliding surface designated by the designers [12]. The system trajectories are then constrained to the sliding surface for all subsequent time via the use of high speed switching controls. The most significant advantage of the sliding mode is robustness against changes in system parameters or disturbances. The major disadvantage associated to the sliding mode control is the chattering phenomena, because it can excite undesirable high frequency dynamics.

The sliding mode control comports three modes, namely, the reaching mode (RM), sliding mode (SM), and steady-state mode (SS).

Let us describe the discrete sliding mode control however, only a few researches are interested by discrete-time systems. A discrete version of SMC has a big importance when the implementation of the control is realized by numerical components which need a sampling period to compute the appropriate controller. It must be pointed out that the discrete version of SMC cannot be obtained from their continuous counterpart by means of simple equivalence. Among the first which are interested by SMC problem and used an equivalent form of the continuous reaching condition to give a discrete reaching condition are Dote and Hoft [5].

$$
[S(k+1)-S(k)] S(k) \prec 0
$$

Milosavljevic [6] recommended the concept of the quasi-sliding mode and signalled hat condition (6) is not sufficient for a discrete sliding mode control. 
Sarpturk, et al. [7], used the following reaching condition.

$$
|S(k+1)| \prec|S(k)|
$$

Furuta [8] used the equivalent form of a Lyapunov-type of continuous reaching condition to give the discrete version.

$$
V(k+1)-V(k) \prec 0 \quad \text { with } V(k)=\frac{1}{2}(S(k))^{2}
$$

Weibing Gao et al. [9] pointed out that all these forms of reaching conditions are incomplete for a satisfactory characterization of a discrete-time sliding mode. He suggests that the state trajectory of a discrete sliding mode control system must have some attributes which form the basis of the discrete sliding mode control, for more information see [9]:

\subsection{Discrete fuzzy sliding mode control law}

For a discrete SMC the following reaching law has been chosen:

$$
S(k+1)=S(k)-q T S(k)-\varepsilon T \operatorname{sgn}(S(k)), \text { with } 1-q T \succ 0, \varepsilon \succ 0, q \succ 0 .
$$

The sliding surface is defined as: $S(k)=G^{T} X(k)$

where $G^{T}$ is a constant row vector $G^{T}=\left[g_{1}, \ldots, g_{n-1}, 1\right]$ such that all the roots of the following polynomial are situated in the left-half open complex plane:

$h(s)=s^{n-1}+g_{n-1} s^{n-2}+\ldots+g_{1}$

The sliding Mode control comports two terms which are:equivalent control term and switching control term[3][5][7][12].

$$
u_{g}=u_{e}+u_{s}
$$

\section{The equivalent control law}

In the first part we assume that : $\Delta A_{d i}=0 n \times n$ and $\Delta B_{d i}=[0, \ldots, 0]^{T}$.

The switching function is defined as: $S(k)=G^{T} X(k)$ The ideal quasi sliding mode satisfies: $\quad S(k+1)=S(k)=0$

We deduct :

$$
0=G^{T} \sum_{i=1}^{c} h_{i}(Z(k))\left(A_{d i}(k+1) X(k)+B_{d i} u_{e}(k)\right) ; k=0,1, \ldots
$$

The equivalent control term is given by:

$$
u_{e}(k)=-\left(\sum_{i=1}^{c} h_{i}(Z(k)) G^{T} B_{d i}\right)^{-1}\left[G^{T} \sum_{i=1}^{c} h_{i}(Z(k)) A_{d i}(k+1) X(k)\right]
$$

We assume that condition is hold: $\left(\sum_{i=1}^{c} h_{i}(Z(k)) G^{T} B_{d i}\right) \neq 0$

\section{The switching control law}

From the reaching law we can write:S(k+1) $-S(k)=-q T S(k)-\varepsilon T \operatorname{sgn}(S(k))$

$$
S(k+1)-S(k)=G^{T} \sum_{i=1}^{c} h_{i}(Z(k))\left(A_{d i}(k+1) X(k)+B_{d i} u(k)\right)-G^{T} X(k)
$$


If we compare the two latest equations we deduct the global control law:

$$
u_{g}(k)=-\digamma^{-1}\left[\sum_{i=1}^{c} h_{i}(Z(k))\left(G^{T} A_{d i}(k+1)\right) X(k)-(1-q T) S(k)+\varepsilon T \operatorname{sgn}(S(k))\right]
$$

where $\digamma=\left(\sum_{i=1}^{c} h_{i}(Z(k)) G^{T} B_{d i}\right)$

From equations and we obtain the switching control term:

$$
u_{S}(k)=-\left(\sum_{i=1}^{c} h_{i}(Z(k)) G^{T} B_{d i}\right)^{-1}[-(1-q T) S(k)+\varepsilon T \operatorname{sgn}(S(k))]
$$

\subsection{Robust fuzzy sliding mode Control law}

Consider the discrete system in the perturbed condition. It will be described by the T-S type fuzzy model. Where $\Delta A_{d i}$ represents system parameters variation and $\Delta B_{d i}$ is the external disturbance for each sub-model. We assume the matching conditions are satisfied: $\Delta A_{d i}=B_{d i} \widetilde{A_{d i}}$ and $\Delta B_{d i}=B_{d i} \breve{B_{d i}}$

where, $A_{d i}$ is a row vector and $B_{d i}$ is a scalar. They should be written as:

$$
\widetilde{A_{d i}}=\left[\begin{array}{llllll}
-\Delta a_{i 1} & -\Delta a_{i 2} & . & . & . & -\Delta a_{i n}
\end{array}\right] \quad \widetilde{B_{d i}}=-\Delta b_{i} \text {. }
$$

Then the equation (5) becomes:

$$
X(k+1)=\sum_{i=1}^{c} h_{i}(Z(k))\left(A_{d i} X(k)+B_{d i} u(k)+\breve{A_{d i} X}(k)+\breve{B_{d i}}\right)
$$

However, the global control law will be expressed as:

$$
u_{g}(k)=-\digamma^{-1}\left[\sum_{i=1}^{c} h_{i}(Z(k))\left(G^{T} A_{d i}(k+1)\right) X(k)+\Gamma-(1-q T) S(k)+\varepsilon T \operatorname{sgn}(S(k))\right]
$$

where $\Gamma=G^{T} B_{d i}\left(\widetilde{A_{d i}} X(k)+\widetilde{B_{d i}}\right)$

In general case and are unknown, but their upper bound are known, so the last global control law can not be implemented. However, to over com this difficulty we replace respectively the unknown terms and by the following expressions:

$$
\begin{aligned}
& \overline{A_{i}}=\left(\sqrt{\left(\operatorname{eig}\left(A_{\text {diup }}^{T} A_{\text {diup }}^{\smile}\right)\right)}\right)^{T} ; \overline{B_{i}}=\Delta b_{\text {imax }} .
\end{aligned}
$$

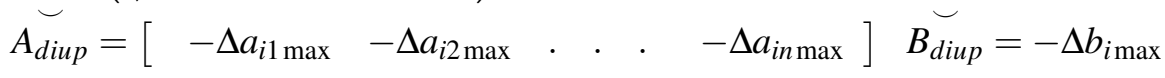

We define a new set of perturbations and control parameters as follow:.

$$
S_{i g}=G^{T} B_{d i} \overline{A_{i}} X(k) ; \quad F_{i g}=G^{T} B_{d i} \overline{B_{i}}
$$

The choice of $S_{i g}$ and $F_{i g}$ is done to ensure that the sign of the incremental $S(k)$ is opposite to the sign of $S(k)$.

The global control law will be expressed as:

$$
u_{g}(k)=-\digamma^{-1}\left[\sum_{i=1}^{c} h_{i}(Z(k))\left(G^{T} A_{d i}\right) X(k)+Q_{i}-(1-q T) S(k)+\varepsilon T \operatorname{sgn}(S(k))\right]
$$

where $Q_{i}=\left(S_{i g}+F_{i g}\right)-\left(S_{i g}+F_{i g}\right) \operatorname{sgn}(S(k))$ 


\subsection{Tracking robust fuzzy sliding mode Control law}

The tracking problem will be transformed into the stability problem. Indeed, $S(k)=0$ represents an equation whose unique stationary solution is . The tracking problem of the desired vector $X_{d}$ comes back to locate inside the quasi-sliding band width the sliding surface for all sampling time. The sliding surface will be expressed as: $S(k)=G^{T} \widetilde{X}(k)$ where, $\widetilde{X}(k)=X(k)-X_{d}(k)$

The control law will be expressed as:

$$
u_{g}(k)=-\digamma^{-1}\left[\sum_{i=1}^{c} h_{i}(Z(k))\left(G^{T} A_{d i}\right) \tilde{X}(k)+Q_{i}-(1-q T) S(k)+\varepsilon T \operatorname{sgn}(S(k))\right]
$$

\section{Illustration}

To illustrate the performance of the presented approach, we choose inverted pendulum and Mass spring damper which are widely used in the control literature of nonlinear system.

\subsection{Inverted pendulum}

The equations of system in continuous form are given by (20) [18]: where, $x_{1}$ is the angle in radian of the pendulum from the vertical axis; $x_{2}$ is the angular velocity in $\mathrm{rad} / \mathrm{s} ; g$ is the gravity acceleration; $m$ and $2 l$ are respectively the mass and the length of the pendulum; $M$ is the mass of the cart and $\mathrm{u}$ is the force applied to the cart. The nominal values of the parameters are: $g=9.81 \mathrm{~m} / \mathrm{s} 2, m=2 \mathrm{~kg}, M=8 \mathrm{~kg}, 2 l$ $=1 \mathrm{~m}$.

$$
\left\{\begin{array}{c}
\dot{x}_{1}(t)=x_{2}(t) \\
\dot{x}_{2}(t)=f\left(x_{1}, x_{2}\right)+g\left(x_{1}, x_{2}\right) u+d(t) \\
f\left(x_{1}, x_{2}\right)=\frac{m l x_{2}^{2} \sin x_{1} \cos x_{1}-(m+M) g \sin x_{1}}{m l \cos ^{2} x_{1}-\frac{4 l}{3}(m+M)} ; g\left(x_{1}, x_{2}\right)=\frac{\cos x_{1}}{m l \cos ^{2} x_{1}-\frac{4 l}{3}(m+M)}
\end{array}\right.
$$

The membership functions for $\left.x_{i} \in\right]-\pi / 2, \pi / 2[$ are:

$$
\mu_{1 i}\left(x_{i}\right)=1-\left|\frac{x_{i}(k)}{\pi / 2}\right| \text { and } \mu_{2 i}\left(x_{i}\right)=\left|\frac{x_{i}(k)}{\pi / 2}\right| \text {, }
$$

The state matrices and input vectors for sub-systems are:

$$
A_{d 1}=\left[\begin{array}{cc}
1 & 0.01 \\
0.1729 & 1
\end{array}\right] ; A_{d 2}=\left[\begin{array}{cc}
1 & 0.01 \\
0.0936 & 1
\end{array}\right], B_{d 1}=\left[\begin{array}{c}
0 \\
0.0018
\end{array}\right], B_{d 2}=\left[\begin{array}{c}
0 \\
0.000052
\end{array}\right]
$$

$\Delta A_{d 1 u p}=\left[\begin{array}{cc}0 & 0 \\ 0.05229 & 0\end{array}\right], ; \Delta A_{d 2 u p}=\left[\begin{array}{cc}0 & 0 \\ 0.028 & 0\end{array}\right], \Delta B_{d 1 u p}=0.0005 ; \Delta B_{d 2 u p}=0.00002$

We have been chosen: $q=70 ; T=0.01 ; \varepsilon=0.1 ; G^{T}=[101]$;

The figure 1 presents the simulation results of the behavior of variable state $x_{1}(k)$ and $S(k)$ of inverted pendulum for nominal system. The initial conditions are given by: $X(0)=[\pi / 3 ; 0]$.

We present by the figures $2,3,4,5,6$ and 7 the simulation results of the behavior of the state variables $x_{1}(k), x_{2}(k)$, the position and velocity error $e_{1}(k)$ and $e_{2}(k)$, the sliding surface and the control law respectively of the inverted pendulum with parameters vary of an uncertain way in time.The initial conditions are given by: $X(0)=[-\pi / 60 ; 0] ; G^{T}=[151], \mathrm{q}=80, \varepsilon=1.5$;

The function sign is replaced by the well known sat function which is defined as:

$$
\left\{\begin{array}{c}
\text { if } S \prec \frac{1}{\Phi} ; \operatorname{sat}=S \\
\text { if } S \succeq \frac{1}{\Phi} ; \operatorname{sat}=\operatorname{sgn}(S)
\end{array}\right.
$$




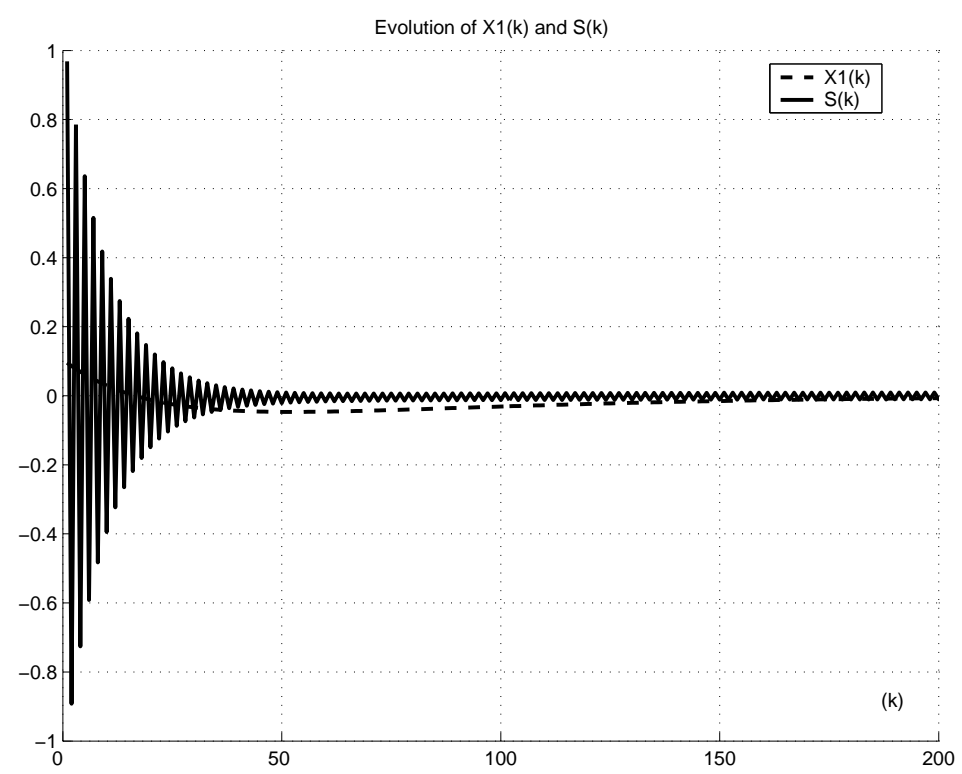

Figure 1: Stabilization of $X l(k)$ and the sliding surface $S(k)$

\subsection{Mass spring damper}

The Mass-spring-damper system is described in continuous time by the following equation [17]: $M_{1} \ddot{x_{1}}(t)+c_{1} x_{1}(t)+c_{2} \dot{x_{1}}(t)+c_{3} x_{1}(t)+c_{4} x_{1}^{2}(t)=\left(1+c 5\left(\dot{x_{1}}\right)^{3}(t)\right) u(t)$

TheT-S fuzzy model is discribe by the following rules:

Rule 1: IF $x_{1}$ is $M_{11}$ and $x_{2}$ is $M_{12}$ THEN $X(k+1)=A_{d 1} X(k)+B_{d i 1} u(k)$

Rule 2: IF $x_{1}$ is $M_{21}$ and $x_{2}$ is $M_{22}$ THEN $X(k+1)=A_{d 2} X(k)+B_{d i 2} u(k)$

Rule 3: IF $x_{1}$ is $M_{31}$ and $x_{2}$ is $M_{32}$ THEN $X(k+1)=A_{d 3} X(k)+B_{d i 3} u(k)$

Rule 4: IF $x_{1}$ is $M_{41}$ and $x_{2}$ is $M_{42} \operatorname{THEN} X(k+1)=A_{d 4} X(k)+B_{d i 4} u(k)$

For nominal values of $M_{1}, c_{2}, c_{3}$ and $c_{4}$, matrices $A_{i}$ and $B_{i}$ are given by:

$$
\begin{aligned}
& A_{d 1}=A_{d 2}=\left[\begin{array}{cc}
1 & 0.01 \\
-0.0001 & 0.99
\end{array}\right] ; A_{d 3}=A_{d 4}=\left[\begin{array}{cc}
1 & 0.01 \\
-0.0023 & 0.99
\end{array}\right] ; \\
& B_{d 1}=B_{d 3}=\left[\begin{array}{c}
0 \\
0.0143
\end{array}\right], B_{d 2}=B_{d 4}=\left[\begin{array}{c}
0 \\
0.0056
\end{array}\right] ; \\
& \Delta A_{d 1 \text { up }}=\Delta A_{d 2 u p}=\left[\begin{array}{cc}
0 & 0 \\
0 & 0.003
\end{array}\right], \Delta A_{d 3 u p}=\Delta A_{d 4 u p}=\left[\begin{array}{cc}
0 & 0 \\
0.0007 & 0.003
\end{array}\right] \\
& \Delta B_{d 1 \text { up }}=\Delta B_{d 3 \text { up }}=0.043 ; \Delta B_{d 2 u p}=\Delta B_{d 4 u p}=0.0017
\end{aligned}
$$

The initial condition and parameters are chosen as: $X(0)=[-\pi / 60 ; 0] ; G^{T}=[151], \mathrm{T}=0.01 ; \mathrm{q}=70, \varepsilon$ $=0.15$.

We present by the figures 8 and 9 the simulation results of the behavior of the state variables $x_{1}(k), x_{2}(k)$, of the mass spring damper with parameters vary of an uncertain way in time.

We present by the figures $10,11,12$ and 13 the simulation results of the behavior of the position and velocity error $e_{1}(k)$ and $e_{2}(k)$, the sliding surface and the control law respectively of the mass spring damper with parameters vary of an uncertain way in time. 


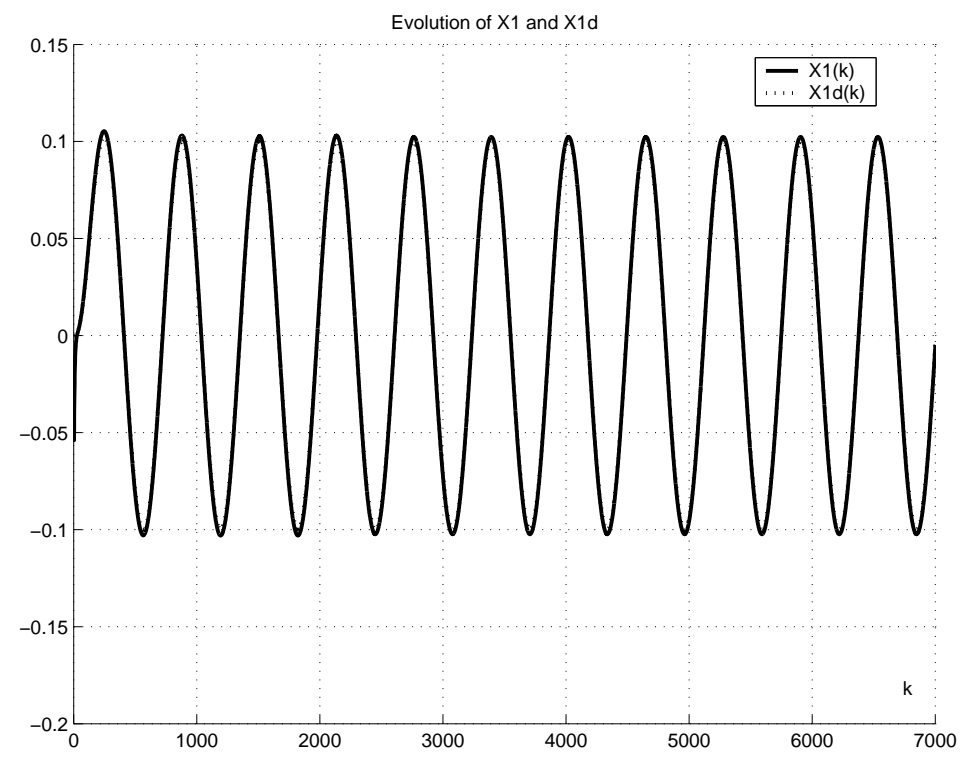

Figure 2: Evolution of $x 1$ and $x l d$

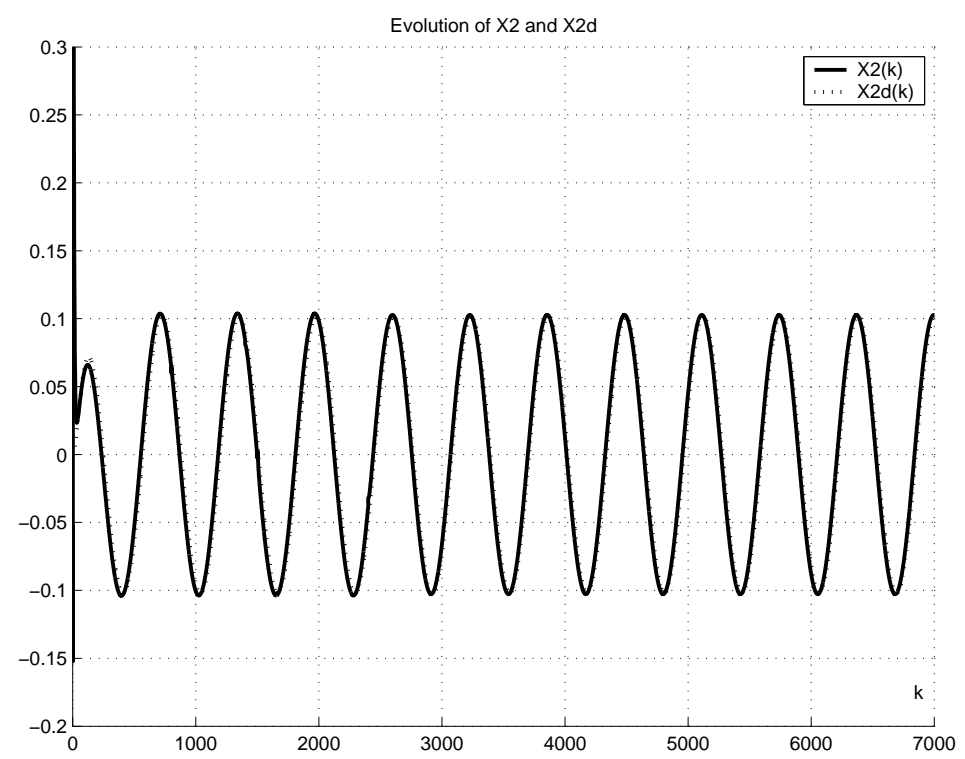

Figure 3: Evolution of $x 2$ and $x 2 d$ 


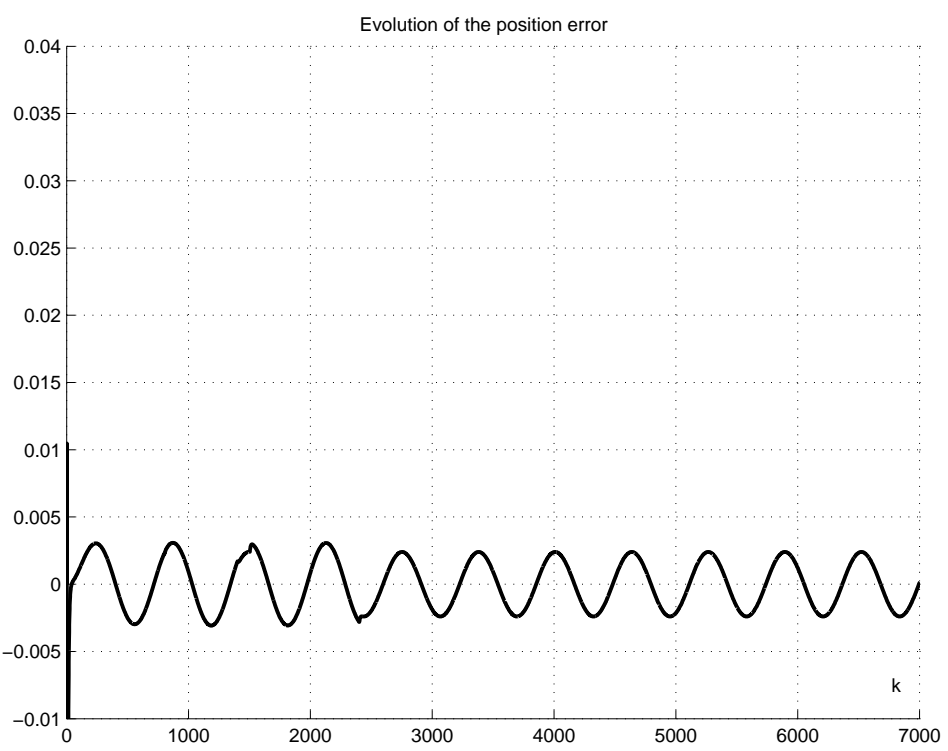

Figure 4: Evolution of the position error

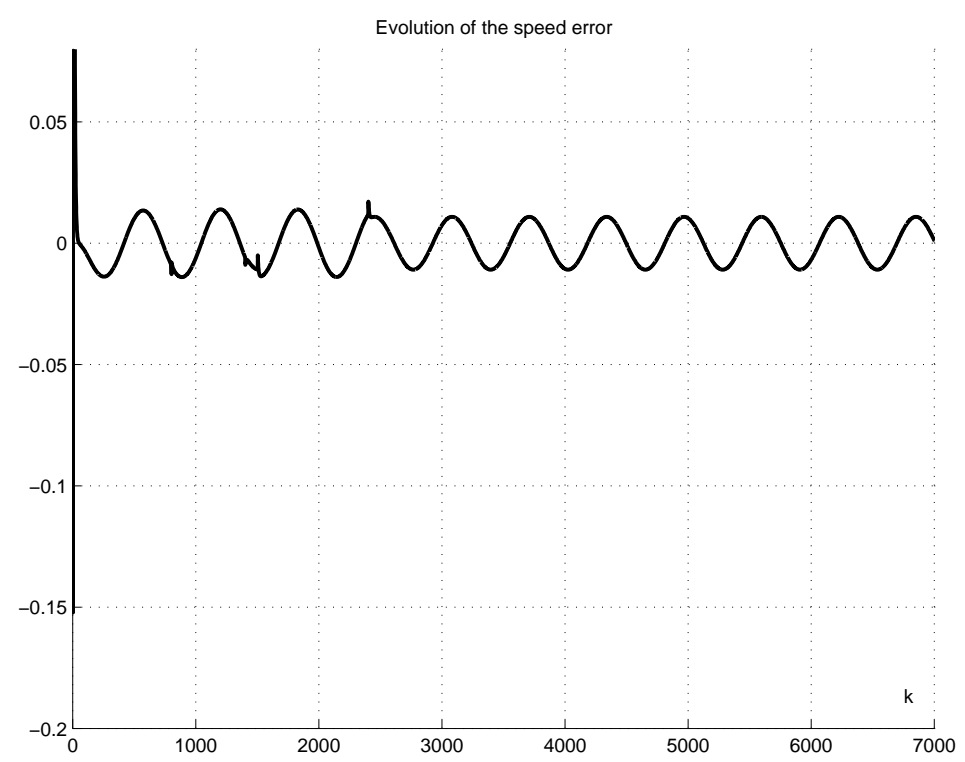

Figure 5: Evolution of the speed error 


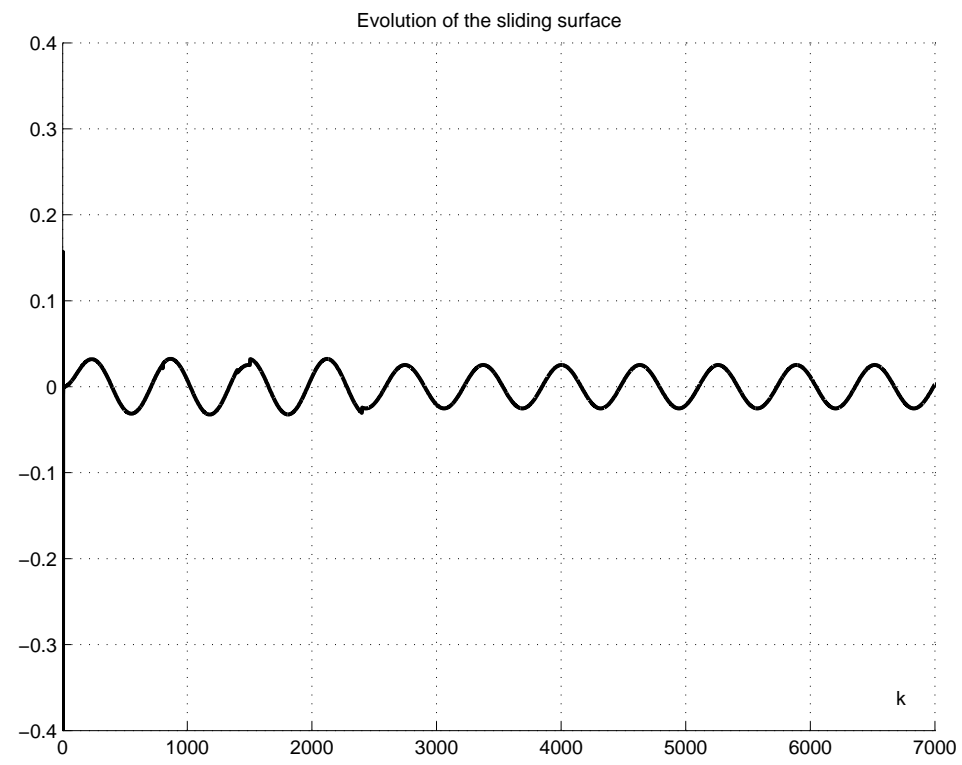

Figure 6: Evolution of the sliding surface

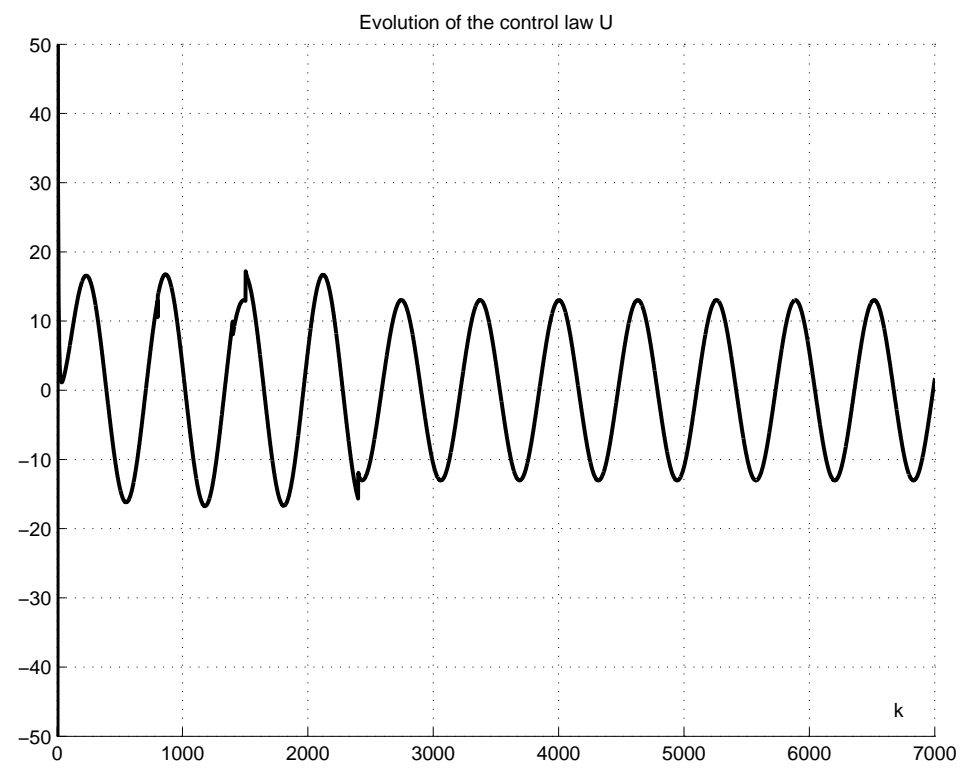

Figure 7: Evolution of the control law 


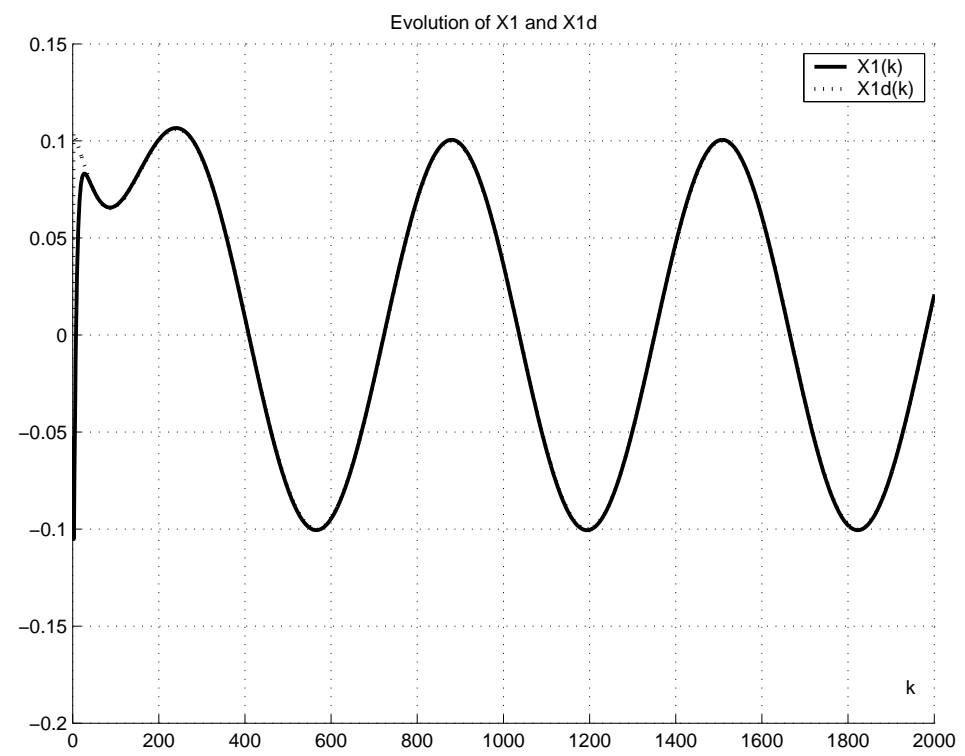

Figure 8: Evolution of $x l$ and $x l d$.

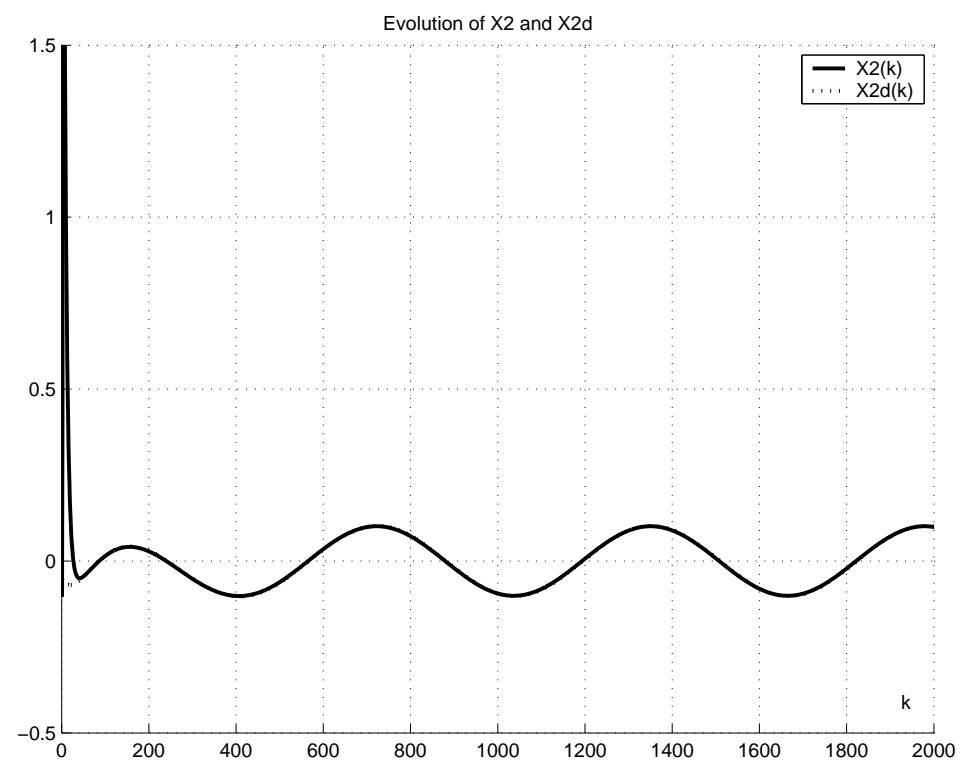

Figure 9: Evolution of $x 2$ and de $x 2 d$. 


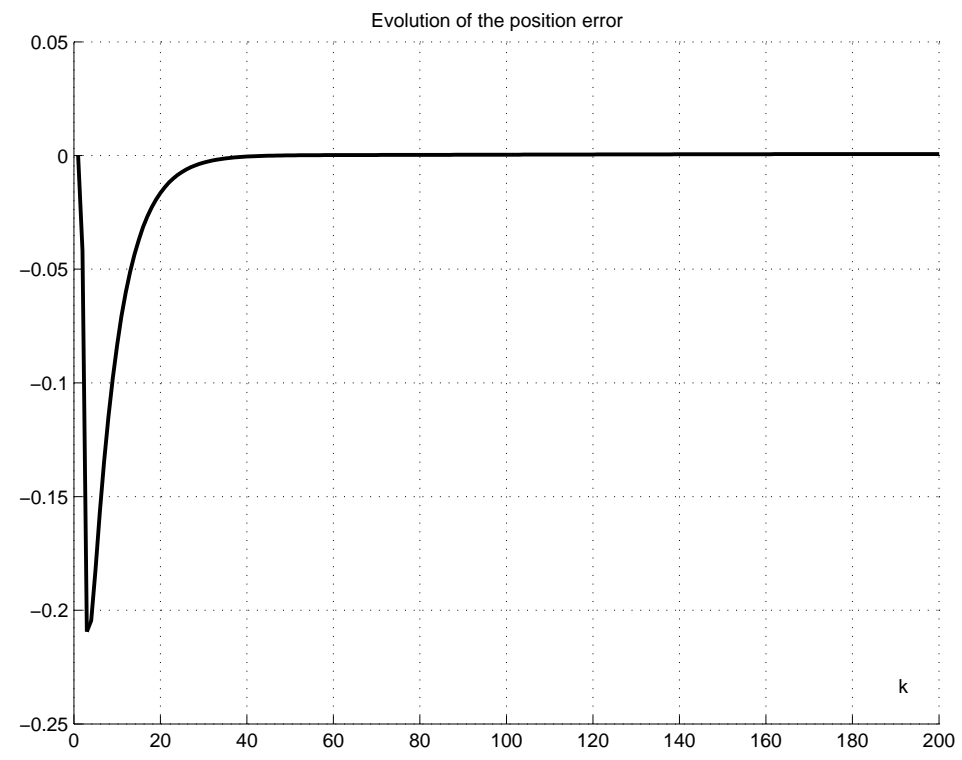

Figure 10: Evolution of the position error

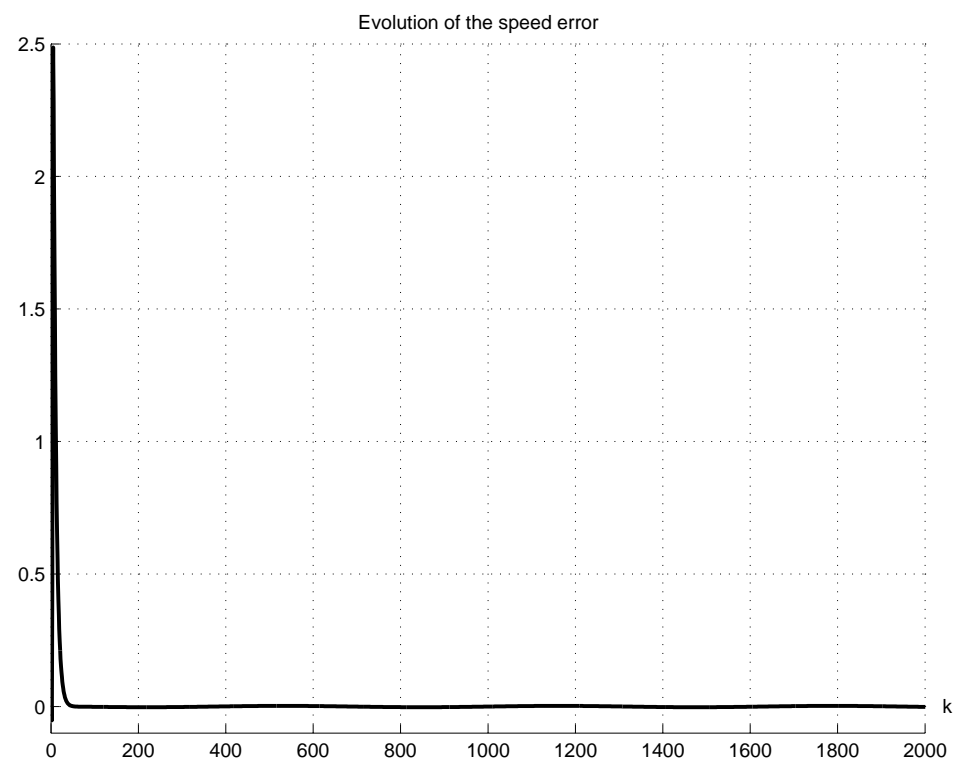

Figure 11: Evolution of the speed error 


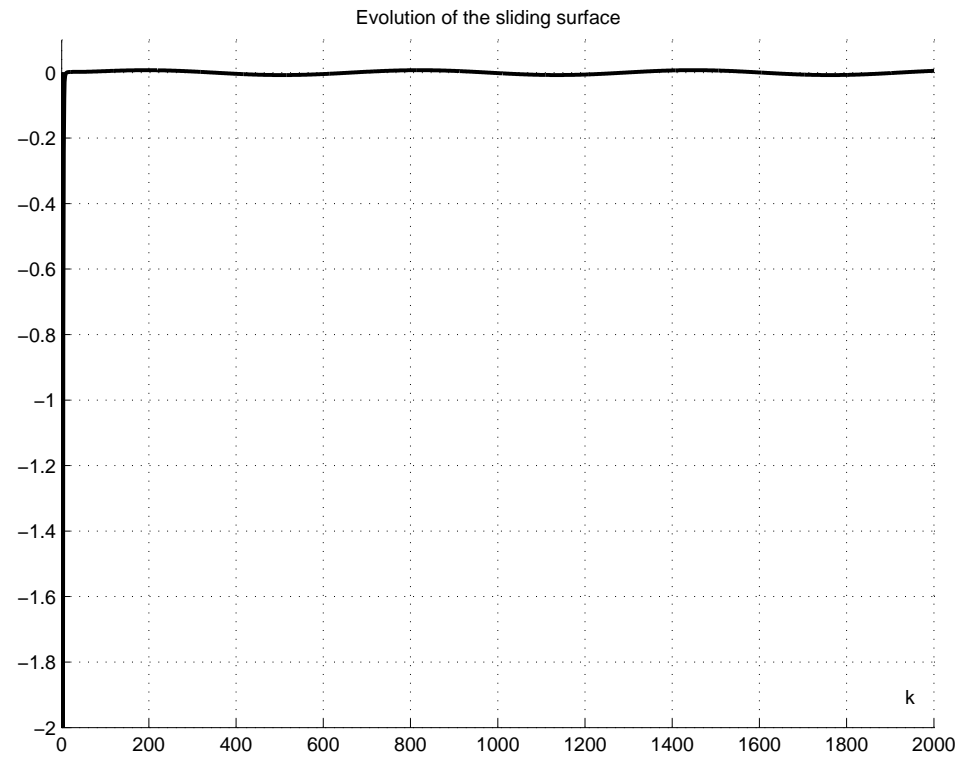

Figure 12: Evolution the sliding surface

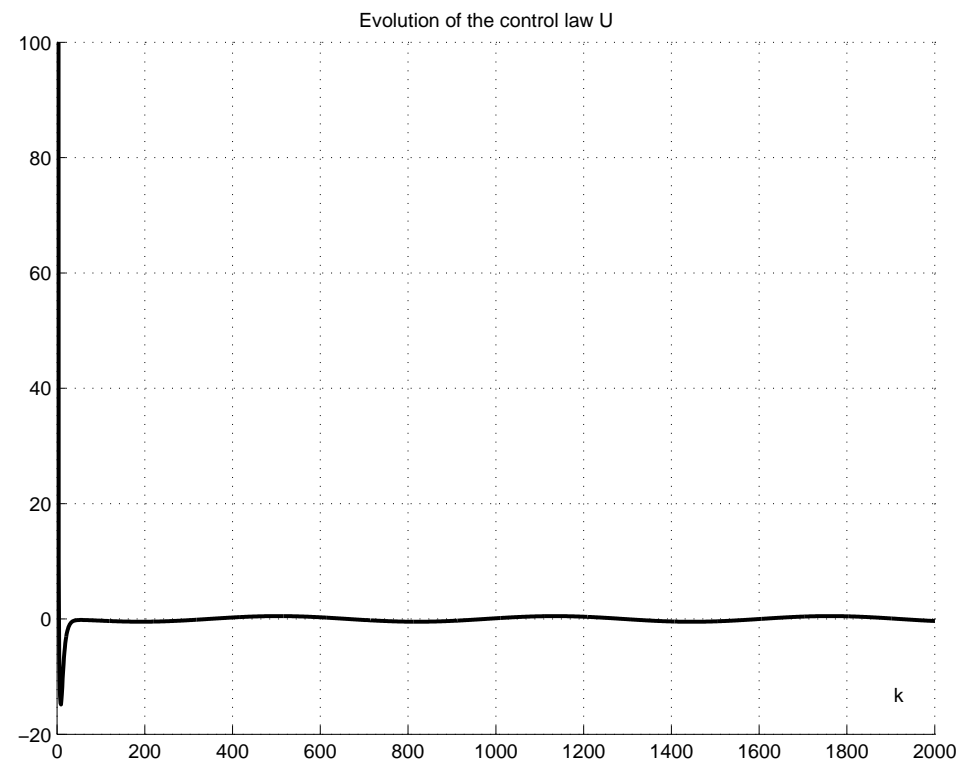

Figure 13: Evolution of the control law 


\section{Conclusions}

In this paper we present a robust fuzzy sliding mode controller for discrete nonlinear systems. First, we recall the discrete Takagi-Sugeno type fuzzy model, then the principe of the sliding mode control in discrete time. The uncertainty are assumed to be verifie the matching conditions. We develop a robust controller based on the sliding mode and the dynamic T-S fuzzy state model. The uncertainties are replaced bye the bigger eigen-value of the upper bound matrices of uncertainties. The expressions in discrete time of both equivalent control term and hitting term are developed. The tracking control law is developed. Simulation results for inverted pendulum and mass spring damper with parameters variation show the performance of the proposed control law.

\section{Bibliography}

[1] Michio Sugeno "On Stability of fuzzy Systems expressed by rules with singleton consequents, (IEEE Transaction on Fuzzy Systems, Vol 7 N 2 Feb 1999).

[2] T.Takagi and M.Sugeno, Fuzzy identification of systems and its applications to modeling and control IEEE trans syst. Man,Cybern. Vol15. 116-132 Jan/Feb1985.

[3] W.Chang, J.Bae Park, Y.Hoan Joob and G.chen Design of robust fuzzy model-based controller with sliding mode control for SISO non-linear systems Fuzzy Sets and Systems 125 (2002) pp 1-22.

[4] Hafedh Abid Mohamed Chtourou et Ahmed Toumi, A Sliding Mode Based Robust Fuzzy Controller For a Class of Uncertain Systems SSD05, Mrach 2005 Sousse Tunisia.

[5] Y. dote and R.G..hoft, Microprocessor based sliding mode controller for dc motor drives presented at the Industrial Application. SOC. Annu. Meeting, Cincinnati, OH, 1980.

[6] D. Milosavljevic, General conditions for the existence of a quasi-sliding mode on the switching hyperplane in Discrete Variable Systems Automat. Remote Contr., vol. 46, pp(307-314) 1985.

[7] S. Z. Sarpturk, Y Istefanopulos, and O. Kaynak, On the stability of Discrete-time sliding mode control systems IEEE Trans. Automat. Contr., vol. 32, N 10, pp(930-932) 1987..

[8] K. Furuta, Sliding Mode Control of a discrete system", Systems and Control Letter, Volume 14, pp145-152.

[9] Weibing Gao, Yufu Wang and Abdollah Homaifa, Discrete-Time Variable Structure Control Systems IEEE Transactions on Industrial, Electronics, volume 42, N 2, April 1995, pp(117-122).

[10] Weibing Gao., and Hung, J.C., Variable structure control of nonlinear systems: a new approach IEEE Transactions on Industrial Electronics, 40, 45, 1993

[11] H. Lee, E. Kim, H.J. Kang et M. Park, A new sliding-mode control with fuzzy boundary layer Fuzzy Sets and Systems 120 (2001), pp. 135-143

[12] Utkin, V. I, Sliding Modes and their Application in Variable Structure Systems (Moscow: Nauka) 1974 (in Russian, and also, 1978, Mir, in English).

[13] Utkin, V. I, Variable structure systems with sliding mode” IEEE Transactions on Automatic Control, Vol. AC-22, No. 2, pp 212-222, April., (1977).

[14] Yu, X. H., Man, Z. H. and Wu, B. L Design of fuzzy sliding-mode control systems, Fuzzy Sets and Systems, 95, pp.295-306. ., (1998) 
[15] Ting, C. S., Li, T. H. S, and Kung, F. C., An approach to systematic design of the fuzzy control system Fuzzy Sets and Systems, 77, pp. 151-166. (1996).

[16] Slotine, J. J. E. and Li. W., Applied nonlinear control, Prentice Hall, Englewood Cliffs, NJ.(1991),

[17] Kazuo Tanaka, Takayuki Ikeda and Hua O.Wang, Robust stabilization of a class of uncertain nonlinear systems via fuzzy control: Quadratic stability, Ho Control Theory, and linear Matrix Inequalities IEEE Trans on Fuzzy System, Vol 4 N 1 Feb 1996.

[18] Mehrdad Hojati and Saeed Gazor Hybrid Adaptive Fuzzy Identification and Control nonlinear SystemsIEEE Transactions ON Fuzzy Systems vol 10, N 2. April 2002 pp 198-210.

Hafedh Abid ${ }^{1,3}$, Mohamed Chtourou ${ }^{2}$, Ahmed Toumi $^{3}$

${ }^{1}$ Institut supérieure des études technologiques de Sfax Laboratoire d'Automatique, Génie Informatique et Signal Cité Scientifique, BP 48, 59651 Villeneuve d'Ascq, France

${ }^{2}$ Unité de Commande Intelligente, design et optimisation des Systèmes complexes(ICOS) ENIS, B.P. W, 3038 sfax, Tunisie

${ }^{3}$ Unité de Procédés Industriels Unité de Commande Automatique (UCPI) ENIS,B.P. W, 3038 sfax, Tunisie

E-mail: Hafedh.abid@isetso.rnu.tn Mohamed.chtourou@enis.rnu.tn Ahmed.toumi@sta-tn.com Received: June 13, 2007 